\title{
Treatment modality and outcomes among early-stage non-small cell lung cancer patients with COPD: a cohort study
}

\author{
Noeul Kang ${ }^{1 \#}$, Sun Hye Shin ${ }^{1 \#}$, Jae Myoung Noh $^{2 \#}$, Danbee Kang ${ }^{3,4}$, Hojoong Kim ${ }^{1}$, O Jung Kwon ${ }^{1}$, \\ Hongryull Pyo ${ }^{2}$, Yong Chan $\mathrm{Ahn}^{2}$, Hong Kwan Kim ${ }^{5}$, Yong Soo Choi ${ }^{5}$, Jhingook Kim ${ }^{5}$, Jae Ill Zo ${ }^{5}$, \\ Young Mog Shim ${ }^{5}$, Juhee Cho ${ }^{3,4,6 *}$, Hye Yun Park ${ }^{1 *}$
}

${ }^{1}$ Division of Pulmonary and Critical Care Medicine, Department of Medicine, Samsung Medical Center, Sungkyunkwan University School of Medicine, Seoul, South Korea; ${ }^{2}$ Department of Radiation Oncology, Samsung Medical Center, Sungkyunkwan University School of Medicine, Seoul, Korea; ${ }^{3}$ Department of Clinical Research Design and Evaluation, SAIHST, Sungkyunkwan University, Seoul, South Korea; ${ }^{4}$ Center for Clinical Epidemiology, Samsung Medical Center, Seoul, South Korea; ${ }^{5}$ Department of Thoracic and Cardiovascular Surgery, Samsung Medical Center, Sungkyunkwan University School of Medicine, Seoul, South Korea; ${ }^{6}$ Department of Epidemiology and Welch Center for Prevention, Epidemiology, and Clinical Research, Johns Hopkins University Bloomberg School of Public Health, Baltimore, MD, USA

Contributions: (I) Conception and design: N Kang, SH Shin, JM Noh, J Cho, HY Park; (II) Administrative support: J Cho, HY Park; (III) Provision of study materials or patients: H Kim, OJ Kwon, H Pyo, YC Ahn, HK Kim, YS Choi, J Kwon, JI Zo, YM Shim, HY Park; (IV) Collection and assembly of data: N Kang, SH Shin, JM Noh, H Kim, OJ Kwon, H Pyo, YC Ahn, HK Kim, YS Choi, J Kim, JI Zo, YM Shim, HY Park; (V) Data analysis and interpretation: N Kang, SH Shin, JM Noh, J Cho, HY Park; (VI) Manuscript writing: All authors; (VII) Final approval of manuscript: All authors.

\#These authors contributed equally to this work.

*These authors contributed equally to this work.

Correspondence to: Hye Yun Park. Division of Pulmonary and Critical Care Medicine, Department of Medicine, Samsung Medical Center, Sungkyunkwan University School of Medicine, 81 Irwon-ro, Gangnam-gu, Seoul, 06351, South Korea. Email: hyeyunpark@skku.edu.

Background: While there is an increasing number of early-stage non-small cell lung cancer (NSCLC) patients with chronic obstructive pulmonary disease (COPD), there are no specific clinical guidelines for treating them. This study aims to evaluate different treatment modalities and corresponding clinical outcomes among early-stage NSCLC patients with COPD.

Methods: We retrospectively reviewed 692 patients with stage I and II NSCLC and COPD from January 2012 to June 2014. Patients were categorized into four groups according to primary treatment modality: surgery only group ( $n=442)$, surgery with adjuvant treatment group ( $n=157)$, radiotherapy (RT) group ( $=48)$, and supportive care (SC)-only group ( $\mathrm{n}=45)$.

Results: Overall, mortality rate was the highest in the SC-only group (35.7 deaths per 100 person-years), followed by RT group (21.5 deaths per 100 person-years), surgery with adjuvant treatment group (8.9 deaths per 100 person-years) and surgery only group (7.2 deaths per 100 person-years). The adjusted hazard ratios (HR) for all-cause mortality compared to the surgery only group were 1.18 (95\% CI, 0.84-1.67) in surgery with adjuvant treatment group, 1.61 (95\% CI, 1.01-2.57) in RT group and 3.23 (95\% CI, 1.99-5.23) in SConly group.

Conclusions: Surgical resection should be considered as the first choice for early-stage NSCLC with COPD. Despite poor lung function or general patient condition, RT rather than SC can be an alternative option if surgery is not feasible. A multi-disciplinary approach and active communication between patients and physicians might be helpful for adequate decision-making regarding treatment for patients with earlystage NSCLC and COPD.

Keywords: Non-small cell lung cancer (NSCLC); chronic obstructive pulmonary disease (COPD); surgery; radiotherapy; supportive care

Submitted Jan 24, 2020. Accepted for publication Aug 03, 2020.

doi: $10.21037 /$ jtd-20-667

View this article at: http://dx.doi.org/10.21037/jtd-20-667

(C) Journal of Thoracic Disease. All rights reserved. 


\section{Introduction}

Surgical resection is generally accepted as the optimal treatment for treating early-stage non-small cell lung cancer (NSCLC) $(1,2)$ with 5 -year survival rates of up to $73 \%$ and $50 \%$ for stage I and II NSCLC, respectively (3). However, not all patients with early-stage NSCLC are surgical candidates due to old age, poor pulmonary function, poor physical function, or comorbidities (4) and when untreated, the outcome of patients with routinely detected early stage NSCLC is a distinctly poor with median survival of 10 months (5).

Chronic obstructive pulmonary disease (COPD) is an established risk factor for the development of lung cancer (6-9). COPD is also the most frequent concomitant $(15.3 \%)$ disease in early-stage NSCLC patients (10) and the prevalence of COPD in newly diagnosed lung cancer cases was six-fold greater than in people without lung cancer regardless of smoking exposure in ever-smokers (11). Moreover, patients with COPD have higher risk of pulmonary complications (PPCs) and poorer survival outcomes after surgical resection than those without COPD (12-15). Thus, pulmonary functions are evaluated to assess the feasibility for lung resection, and radiation therapy including stereotactic body radiotherapy (SBRT) is alternatively suggested for the treatment of the earlystage NSCLC, when patients are medically inoperable $(16,17)$. Indeed, radiotherapy (RT) had shown better survival compared to those with supportive care alone $(18,19)$. However, some COPD patients are reluctant to receive either surgery or RT due to cancer stigma, despite being diagnosed with early-stage, treatable lung cancer $(20,21)$.

Recent guidelines from the American Society of Clinical Oncology and the American Society of Radiation Oncology emphasize the importance of timely treatment and shared decision-making in the management of early-stage NSCLC $(22,23)$. While there are increasing number of early-stage NSCLC patients with COPD, there are no specific clinical treatment guidelines for them. Therefore, this real-world study aimed to investigate treatment modalities that earlystage NSCLC patients with COPD received and to evaluate the clinical outcomes based on treatment modalities.

\section{Methods}

\section{Study population}

This is a retrospective cohort study conducted with stage I and II NSCLC patients who were 40 years or older and who had spirometry tests at the time of cancer diagnosis in Samsung Medical Center, Seoul, Korea from January 2012 to June 2014. As our study objective was to evaluate treatment modalities among early stage NSCLC patients with COPD, we selected 703 subjects who had COPD [defined as pre-bronchodilator forced espiratory volue in 1 second $(\mathrm{FEV} 1) /$ forced vital capacity $(\mathrm{FVC})<0.70$ ] at the time of cancer diagnosis. Patients who were diagnosed with interstitial lung disease ( $\mathrm{n}=9$ ), had history of lung cancer $(n=1)$, and were transferred to other hospitals for further treatment $(n=1)$ were excluded. The final study sample included 692 subjects. This study was conducted in accordance with the Declaration of Helsinki (as revised in 2013). The Institutional Review Board of Samsung Medical center approved this study (No. 2018-07-057) and waived the informed consent as we only used de-identified data retrieved from electronic medical records (EMR).

\section{Measurements}

Spirometry and diffusing capacity of the lungs for carbon monoxide (DLco) were performed using the Vmax 22 system (SensorMedics, Yorba Linda, CA, USA) according to criteria established by the American Thoracic Society/ European Respiratory Society $(24,25)$. Absolute values of $\mathrm{FVC}, \mathrm{FEV}_{1}$, and DLco were obtained, and the percentage of the predicted values (\% pred) were calculated using data obtained with a representative Korean sample $(26,27)$. The severity of COPD was staged by the Global Initiative for Chronic Obstructive Lung Disease (GOLD); mild ( $\mathrm{FEV}_{1}$ $\geq 80 \%$ pred), moderate $\left(50 \% \leq \mathrm{FEV}_{1}<80 \%\right.$ pred), severe $\left(30 \% \leq \mathrm{FEV}_{1}<50 \%\right.$ pred), or very severe $\left(\mathrm{FEV}_{1}<30 \%\right.$ pred $)$ (25). Low DLco was defined as DLco $<80 \%$ pred (28).

\section{Stage of NSCLC}

Stage of NSCLC was determined according to the seventh edition of TNM classification (29). NSCLC included adenocarcinoma, squamous cell carcinoma, and other types such as large cell carcinoma, neuroendocrine carcinoma, poorly- or undifferentiated carcinoma, and pleomorphic sarcomatoid carcinoma. Some patients who could not undergo histologic confirmation were included, when the diagnosis of lung cancer was unequivocal based on clinical and image findings.

\section{Treatment modality and outcome}

Patients were primarily grouped into ‘surgery group' or 
'no surgery (NS) group' according to whether patients received curative surgery or not. The surgery group was further categorized as 'surgery only group' for patients who received surgery only and 'surgery with adjuvant treatment group' for patients who received adjuvant chemotherapy and/or RT after surgical resection. The NS group included patients who received definitive RT (RT group) and patients who did not receive any definitive treatment for lung cancer including surgery or definitive RT (supportive care only group, SC-only group). Two investigators (NK and SS) reviewed patient medical records to determine the decision-making process behind treatment decisions for the NS group. When there was any discordance between the reviewers, the study team reviewed those cases and made the final decision. The primary outcome was all-cause mortality, calculated by total number of deaths divided by the personyear (summation of follow-up periods of each person). Person-time was calculated from the first date of treatment (surgery group and RT group) or the date of diagnosis (SConly group) to the date of death, last date known to be alive, or the end of the study period (April 23,2018), whichever comes first. All dates of patient deaths were ascertained from the medical records or the death records from Statistics Korea. In addition, we obtained the following data from the EMR: demographic, behavioral, and other clinical information including age at diagnosis, sex, smoking status, body mass index (BMI), Eastern Cooperative Oncology Group (ECOG) performance status at diagnosis, comorbid conditions, tumor histology, and hemoglobin level that would be associated with either exposure or outcome.

\section{Statistical analysis}

Survival curves were generated by the Kaplan-Meier product-limit method and compared by log-rank tests. We calculated hazard ratios (HR) with $95 \%$ confidence intervals (CI) for all-cause mortality using the Cox proportional hazards regression models. We adjusted for age (continuous), sex (male or female), smoking status (never, or ever), BMI (continuous), performance status (ECOG $\leq 1$, or $\geq 2$ ), comorbidity (none, or any), tumor stage (clinical stage I or II), hemoglobin level (continuous), FEV1 \% predicted and DLco \% pred (continuous) to account for potential confounding factors at the time of NSCLC diagnosis. To improve comparability of the patients in different treatment modality, additional analyses were performed limiting to patients with age $<70$ or $\geq 70$ and patients with clinical stage I. We examined the proportional hazards assumption using plots of the $\log (-\log )$ survival function and Schoenfeld residuals. All tests were two-sided, and $\mathrm{P}$ values $<0.05$ were considered significant. All analyses were performed using Stata software (ver. 14.0; Stata Corporation, College Station, TX, USA).

\section{Results}

\section{Patient characteristics}

The mean (SD) age of the total study population was 67.9 (7.7) years and $87.7 \%$ of patients were male. Of the total, $63.9 \%, 22.7 \%, 6.9 \%$, and $6.5 \%$ patients received surgery only, surgery with adjuvant treatment, RT, or SC-only, respectively (Table 1). The mean (SD) age of surgery only, surgery with adjuvant treatment, RT, and SC-only was 68.0 (7.4) years, $64.9(6.7)$ years, $72.8(9.5)$ years and $73.1(6.8)$ years, respectively. Compared with patients who received surgery regardless of adjuvant therapy, patients in RT and SC-only group were older $(\mathrm{P}<0.001)$ and more likely to be ever-smokers $(\mathrm{P}=0.007)$, and have lower $\mathrm{BMI}(\mathrm{P}<0.001)$. The surgery group had better ECOG performance than RT or SC-only group (ECOG performance status of $0-1,99.3 \%$ in surgery only group, and $98.1 \%$ in surgery with adjuvant treatment group $v s .68 .8 \%$ in RT group and $77.8 \%$ in SC-only group, $\mathrm{P}<0.001)$. Previous history of other cancer was mostly found in RT group $(25.0 \%$, $\mathrm{P}=0.002$ ). Adenocarcinoma was common in surgery only group $(56.6 \%)$ while other groups were mostly consisted of squamous cell carcinoma $(60.5 \%$ in surgery with adjuvant treatment group, $54.2 \%$ in RT group and $57.8 \%$ in SC-only group, $\mathrm{P}<0.001)$. Surgery with adjuvant treatment group had more clinical stage II (62.4\%) than other groups (23.8\% in surgery only, $33.3 \%$ in RT group and $40.0 \%$ in SC-only group, $\mathrm{P}<0.001)$. Among the RT group, 15 patients received SBRT. The clinical characteristics of the SC-only group were not different from those of the RT group (Table S1). Compared to the surgery group, the NS group had significantly poor lung function in terms of $\mathrm{FVC}$, $\mathrm{FEV}_{1}, \mathrm{FEV}_{1} / \mathrm{FVC}$, and DLco (Table 2). In terms of COPD severity, while only $3.8 \%$ of the surgery group had severe-to-very severe COPD, $33.3 \%$ of the RT and $28.9 \%$ of the SC-only group had severeto-very severe COPD, respectively (Figure 1). Prevalence of reduced DLco ( $<80 \%$ pred) was $51.8 \%$, $55.5 \%, 76.7 \%$, and $63.4 \%$ in the surgery only, surgery with adjuvant treatment, RT, or SC-only group, respectively (Figure 2). 
Table 1 Baseline characteristics of 692 patients with early-stage NSCLC and COPD

\begin{tabular}{|c|c|c|c|c|c|}
\hline & Surgery only ( $\mathrm{n}=442$ ) & Surgery with adjuvant treatment $(n=157)$ & $\mathrm{RT}(\mathrm{n}=48)$ & SC-only $(n=45)$ & $P$ values \\
\hline Male sex & $380(86.0)$ & $141(89.8)$ & $43(89.6)$ & $43(95.6)$ & 0.203 \\
\hline Smoking status ${ }^{\dagger}$ & & & & & 0.007 \\
\hline Current smoker & $162(36.7)$ & $77(49.0)$ & $15(31.3)$ & $19(42.2)$ & \\
\hline Never smoker & $77(17.4)$ & $15(9.6)$ & $4(8.3)$ & $2(4.4)$ & \\
\hline BMI $\left(\mathrm{kg} / \mathrm{m}^{2}\right)^{\dagger}$ & $23.7(2.7)$ & $24.0(2.9)$ & $22.3(3.4)$ & $21.7(3.1)$ & $<0.001$ \\
\hline ECOG Performance Status ${ }^{\ddagger}$ & & & & & $<0.001$ \\
\hline $0-1$ & 439 (99.3) & $154(98.1)$ & $33(68.8)$ & $35(77.8)$ & \\
\hline History of other cancer & $83(18.8)$ & $11(7.0)$ & $12(25.0)$ & $6(13.3)$ & 0.002 \\
\hline Double primary cancer & $40(9.1)$ & $8(5.1)$ & $4(8.3)$ & $4(8.9)$ & 0.451 \\
\hline \multicolumn{6}{|l|}{ Comorbidities } \\
\hline Tuberculosis & $62(14.0)$ & 25 (15.9) & $13(27.1)$ & $9(20.0)$ & 0.100 \\
\hline Heart disease & $52(11.8)$ & $17(10.8)$ & $8(16.7)$ & $9(20.0)$ & 0.294 \\
\hline Hypertension & $199(45.0)$ & $63(40.1)$ & $20(41.7)$ & $20(44.4)$ & 0.748 \\
\hline Diabetes & $104(23.5)$ & $24(15.3)$ & $11(22.9)$ & $6(13.3)$ & 0.090 \\
\hline Hemoglobin $(\mathrm{g} / \mathrm{dL})^{\dagger}$ & $12.9(1.4)$ & $12.8(1.5)$ & $12.8(1.4)$ & $13.0(1.6)$ & 0.701 \\
\hline Clinical stage & & & & & $<0.001$ \\
\hline I & 337 (76.2) & $59(37.6)$ & $32(66.7)$ & $27(60.0)$ & \\
\hline II & 105 (23.8) & $98(62.4)$ & 16 (33.3) & $18(40.0)$ & \\
\hline
\end{tabular}

Values are in mean (standard deviation) or number (\%). ${ }^{\dagger}$ The number of patients with missing value was smoking status ( $\left.n=2\right)$, BMl $(n=4)$, and hemoglobin $(n=3)$. ${ }^{\ddagger}$ Performance status was based on Eastern Cooperative Oncology Group (ECOG) scale. BMI, body mass index; COPD, chronic obstructive pulmonary disease; ECOG PS, Eastern Cooperative Oncology Group Performance Status; NSCLC, non-small cell lung cancer; RT, radiotherapy; SC, supportive care.

\section{All-cause mortality according to the treatment modality}

During the median follow up of 4.3 year (interquartile range 2.7-5.1 years; 2,714 person-years), 257 patients died. During the first 90 days, 16 patients (16/692, 2.31\%) died and all of them were patients in the surgery group.
Overall, mortality rate was the highest in the SC-only group (35.7 deaths per 100 person-years), followed by RT group (21.5 deaths per 100 person-years), surgery with adjuvant treatment group (8.9 deaths per 100 personyears) and surgery only group (7.2 deaths per 100 personyears) (Table 3 and Figure 3). After adjusting for age, sex, 
Table 2 Lung function of study participants at the time of NSCLC diagnosis

\begin{tabular}{|c|c|c|c|c|c|}
\hline & Surgery only $(n=442)$ & Surgery with adjuvant treatment $(n=157)$ & $\mathrm{RT}(\mathrm{n}=48)$ & SC-only $(n=45)$ & $P$ values \\
\hline FVC (L) & $3.67(0.8)$ & $3.80(0.7)$ & $3.07(0.7)$ & $3.14(0.7)$ & $<0.001$ \\
\hline FVC (\%predicted) & $89.5(13.1)$ & $90.7(13.6)$ & $78.8(15.3)$ & $79.3(15.7)$ & $<0.001$ \\
\hline $\mathrm{FEV}_{1}(\mathrm{~L})$ & $2.23(0.6)$ & $2.31(0.5)$ & $1.68(0.5)$ & $1.69(0.6)$ & $<0.001$ \\
\hline $\mathrm{FEV}_{1}(\%$ predicted $)$ & $75.9(15.0)$ & $75.4(14.3)$ & $65.1(22.6)$ & $62.5(21.9)$ & $<0.001$ \\
\hline $\mathrm{FEV}_{1} / \mathrm{FVC}$ & $60.7(8.0)$ & $60.8(6.9)$ & $55.1(11.5)$ & $53.0(12.7)$ & $<0.001$ \\
\hline $\mathrm{DLco}(\mathrm{mL} / \mathrm{min} / \mathrm{mmHg})^{\dagger}$ & $16.0(4.4)$ & $16.2(3.8)$ & $11.7(3.0)$ & $13.0(4.3)$ & $<0.001$ \\
\hline DLco $(\% \text { predicted })^{\dagger}$ & $79.7(18.4)$ & $77.9(16.4)$ & $62.9(16.7)$ & $70.4(20.9)$ & $<0.001$ \\
\hline
\end{tabular}

Values are in mean (standard deviation). ${ }^{\dagger}$ Data regarding DLco (L) and DLco \% predicted were available in 675 patients. COPD, chronic obstructive pulmonary disease; DLco, diffusing capacity for carbon monoxide; FEV $_{1}$, forced expiratory volume in $1 \mathrm{~s}$; FVC, forced vital capacity; NSCLC, non-small cell lung cancer; RT, radiotherapy; SC, supportive care.
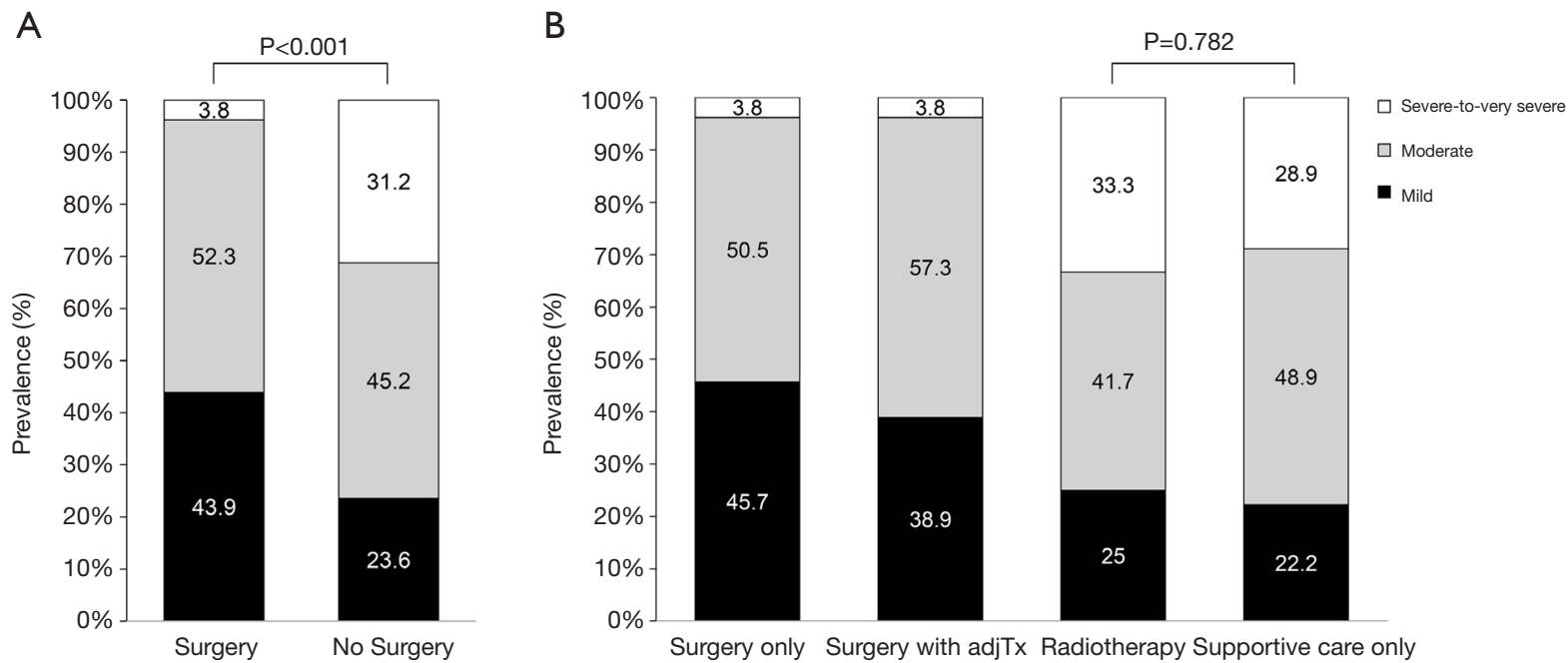

Figure 1 The distribution of COPD severity between (A) surgery vs. no surgery groups and (B) by treatment modalities. COPD, chronic obstructive pulmonary disease.

smoking status, BMI, performance status, comorbidity, hemoglobin level, tumor stage, $\mathrm{FEV}_{1}$ (\% pred), and DLco (\% pred), the adjusted hazard ratios (aHR) for all-cause mortality compared to the surgery only group were 1.18 (95\% CI, 0.84-1.67) in surgery with adjuvant treatment group, 1.61 (95\% CI, 1.01-2.57) in RT group and 3.23 (95\% CI, 1.99-5.23) in SC-only group. Compared to surgery group, adjusted HR of NS group was 2.04 (95\% CI, 1.41-2.96). Among NS group, when outcomes between RT and SC-only group were compared, the SC-only group had significantly higher risk of death compared to RT (aHR, 2.99; 95\% CI, 1.51-5.94).

Considering that there is significant difference of age among patients with different treatment modalities, we performed a subgroup analysis with age $<70$ and $\geq 70$ years (Table S2). However, there was no interaction with age and the results remained as same. When we restricted the analysis with clinical stage I patients, RT group had worse survival compared to the surgery only group (HR, 2.40; 95\% CI, 1.31-4.42), but had better survival than SC-only group (HR in SC-only group compared to RT group, 3.15; 95\% CI, 1.13-8.80) (Table S3).

\section{Discussion}

In this large cohort study of early stage NSCLC patients with COPD, most patients underwent surgical resection only $(63.9 \%)$ or surgical resection with adjuvant treatment 

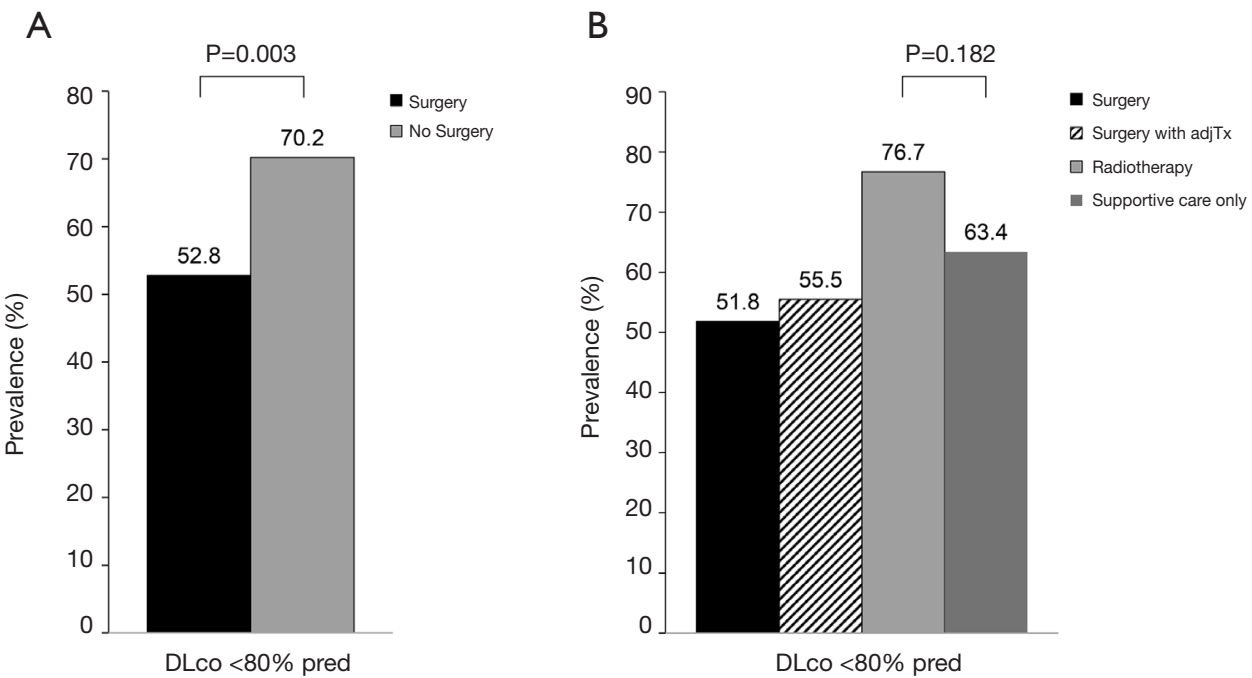

Figure 2 Prevalence of reduced DLco ( $<80 \%$ predicted) between (A) surgery vs. no surgery groups and (B) by treatment modalities. DLco, diffusing capacity for carbon monoxide.

Table 3 Hazard ratios (95\% confidence intervals) for all-cause mortality according to the treatment modality

\begin{tabular}{|c|c|c|c|c|}
\hline & No. of death & Incidence rate per 100 -person year & Crude, HR $(95 \% \mathrm{Cl})$ & Adjusted $^{\dagger}, \mathrm{HR}(95 \% \mathrm{Cl})$ \\
\hline Surgery $(n=599)$ & 189 & 7.7 & Reference & Reference \\
\hline No surgery $(n=93)$ & 68 & 27.2 & $3.52(2.66,4.66)$ & $2.04(1.41,2.96)$ \\
\hline \multicolumn{5}{|l|}{ Specific treatment modality } \\
\hline $\begin{array}{l}\text { Surgery with adjuvant treatment } \\
(\mathrm{n}=157)\end{array}$ & 57 & 8.9 & $1.23(0.90,1.67)$ & $1.18(0.84,1.67)$ \\
\hline $\mathrm{RT}(\mathrm{n}=48)$ & 32 & 21.5 & $2.95(2.00,4.35)$ & $1.61(1.01,2.57)$ \\
\hline SC only $(n=45)$ & 36 & 35.7 & $4.90(3.37,7.12)$ & $3.23(1.99,5.23)$ \\
\hline
\end{tabular}

${ }^{\dagger}$ Adjusted for age, sex, smoking status. BMI, performance status, any comorbidity, hemoglobin level, tumor stage, FEV $\%$ pred and DLco $\%$ pred. $\mathrm{Cl}$, confidence interval; HR, hazard ratio; RT, radiotherapy; SC, supportive care; $\mathrm{FEV}_{1}$, forced expiratory volume in $1 \mathrm{~s}$; DLco, diffusing capacity for carbon monoxide.

(22.7\%) while relatively few received RT $(6.9 \%)$ or SC-only (6.5\%). After adjusting for confounders, patients without surgery had two times higher risk of death compared to patients with surgery, and among patients without surgery, patients who only received SC had about three times higher risk of death compared to patients with RT. These outcomes were consistently observed in clinically relevant subgroups by age and clinical stage. As early curative surgical treatment affects the subsequent treatment, we separated surgery patients with adjuvant treatment from patients with surgery only, and the risk of death were not significantly different between two groups.

In our observational study, however, patients who received surgery and those who did not are a different population with a distinct entity. This is the reflection of the real-world practice of early-staged NSCLC patients with COPD. The surgery group had relatively mild COPD and better general condition, leading to a favorable outcome, compared with NS group. Whereas, the NS group were older, and had more advanced COPD with poor performance status, indicating that those were deemed medically inoperable. Among the patients who did 


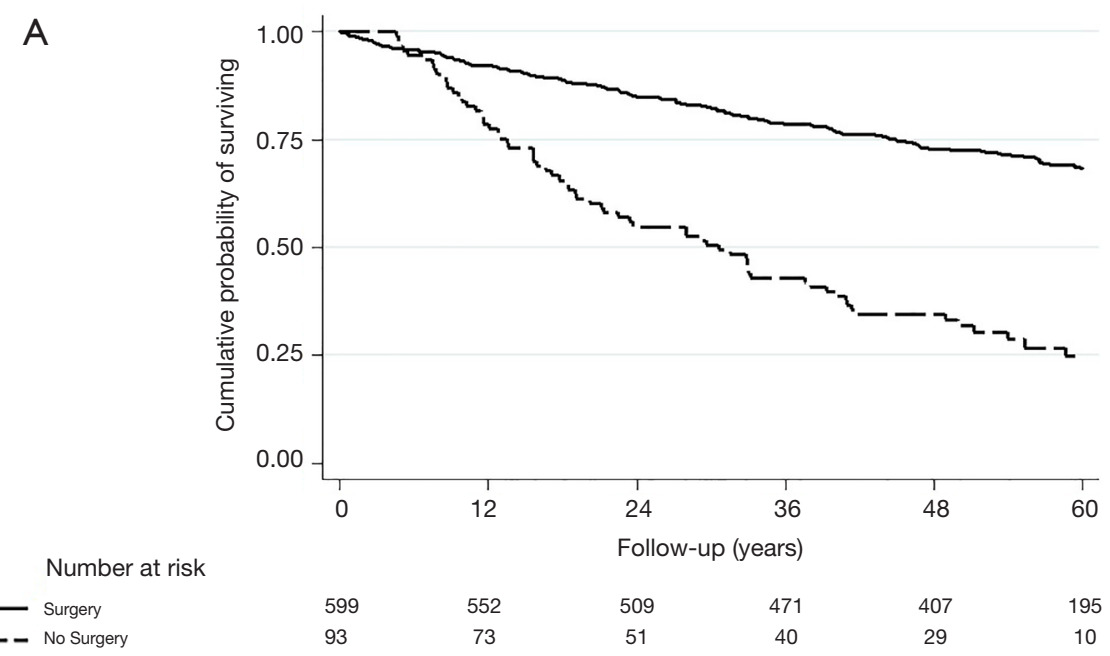

B

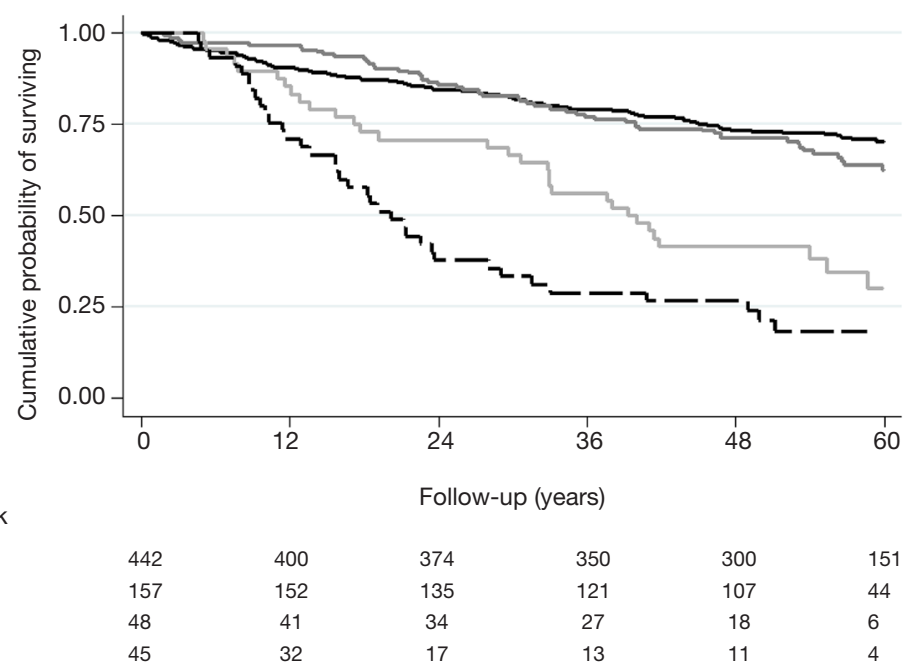

Figure 3 Cumulative probability of surviving between (A) surgery vs. no surgery groups and (B) by treatment modalities.

not undergo surgery, only half of them received RT as an alternative option and the other half of them did not receive curative treatment. The RT group showed significantly better survival than the SC group despite similar age, smoking status, BMI, comorbidities, and lung function between the RT group and SC group. Indeed, previous study demonstrated that definitive radiotherapy was well tolerated in early-stage NSCLC patients with COPD (30). Thus, RT should be taken into account than supportive care only for better survival outcomes in COPD patients who are not feasible to undergo surgical resection.

Our study confirmed that surgical resection is the primary and preferred treatment option for early-stage NSCLC patients with COPD when the COPD patients are feasible to undergo surgical resection, which was consistent with the general guidelines for treating early-stage NSCLC (1). In our study, however, the surgery group had higher postoperative mortality than the NS group during the first 90 days (Figure $3 A$ ), which is in accordance with existing literature documenting the risks of surgery $(31,32)$. Thus, clinicians should be aware of postoperative mortality risk and evaluate patient lung function and overall profiles carefully when considering lung resection surgery for earlystage NSCLC patients with COPD. 
In terms of treatment decision, RT was mostly decided by physicians (concerned primarily with poor lung function) whereas SC-only was mostly decided by patients and family in accordance with their preference not to have active treatment (Table S4). We reviewed medical records to identify key factors used to support the decision to pursue surgical or non-surgical interventions. Some factors that lead to non-surgical intervention were old age, poor lung function, comorbid disease, performance status, and patient will. These factors are in line with the findings from the prospective cohort study of early-stage NSCLC patients (33). In our study, the decision to rely exclusively on SC was typically made by the patient or family with the main reason for not receiving any other definitive treatment including RT being 'patient and family will.' One reason patients and families might decide against treatment is the stigma associated with cancer (20). Despite increasing survival rates, many still consider cancer to be a death sentence and are unwilling to consider any treatment options (34). Furthermore, patients might not have enough information about alternative treatment options such as RT or they might worry too much about treatment complications. Considering that patients in the SC-only group had similar lung function and performance status, more tailored education would be necessary for early-stage NSCLC patients with advanced COPD at diagnosis. In addition, clinicians need to encourage shared decision-making by providing accurate therapeutic information.

There are several limitations of this study. First, since COPD was based on a pre-bronchodilator spirometry (35), the diagnosis of COPD might be overestimated. Second, this study was conducted with patients at a tertiary hospital and the results of the study might not be generalizable to patients at different settings. Thirdly, because this is a retrospective cohort study, we were not able to include all influencing factors which affect the outcomes. Further studies are necessary to confirm our finding. In addition, there might be some information we missed regarding treatment decisions because we only included information available on the EMR and there might be other reasons physicians or patients decide on specific treatments.

\section{Conclusions}

While there are many epidemiologic studies showing the high prevalence of COPD in patients with NSCLC patients, there is limited evidence in terms of treatment modalities and its associated clinical outcomes for lung cancer patients with COPD. The results of our study suggest that surgical resection should be considered as the first choice for earlystage NSCLC with COPD. RT can be an alternative option if surgery is not feasible due to poor lung function or general patient condition. A multi-disciplinary approach for treating early lung cancer patients with COPD is necessary. In addition, more active communication between patients and physicians might be helpful for adequate decisionmaking regarding treatment for patients with early-stage NSCLC and COPD.

\section{Acknowledgments}

Funding: This work was supported by the National Research Foundation of Korea (NRF) grant funded by the Korea government (MSIP) (no. 2017R1A2B2006435) and the Ministry of Science and ICT (No. NRF2017M2A2A7A02018569).

\section{Footnote}

Data Sharing Statement: Available at http://dx.doi. org/10.21037/jtd-20-667

Conflicts of Interest: All authors have completed the ICMJE uniform disclosure form (available at http://dx.doi. org/10.21037/jtd-20-667). The authors have no conflicts of interest to declare.

Ethical Statement: The authors are accountable for all aspects of the work in ensuring that questions related to the accuracy or integrity of any part of the work are appropriately investigated and resolved. This study was conducted in accordance with the Declaration of Helsinki (as revised in 2013). The Institutional Review Board of Samsung Medical center approved this study (No. 2018-07-057) and waived the informed consent as we only used de-identified data retrieved from electronic medical records (EMR).

Open Access Statement: This is an Open Access article distributed in accordance with the Creative Commons Attribution-NonCommercial-NoDerivs 4.0 International License (CC BY-NC-ND 4.0), which permits the noncommercial replication and distribution of the article with the strict proviso that no changes or edits are made and the original work is properly cited (including links to both the formal publication through the relevant DOI and the license). See: https://creativecommons.org/licenses/by-nc-nd/4.0/. 


\section{References}

1. Howington JA, Blum MG, Chang AC, et al. Treatment of stage I and II non-small cell lung cancer: Diagnosis and management of lung cancer, 3rd ed: American College of Chest Physicians evidence-based clinical practice guidelines. Chest 2013;143:e278S-e313S.

2. Ettinger DS, Wood DE, Aisner DL, et al. Non-Small Cell Lung Cancer, Version 5.2017, NCCN Clinical Practice Guidelines in Oncology. J Natl Compr Canc Netw 2017;15:504-35.

3. Tanoue LT, Detterbeck FC. New TNM classification for non-small-cell lung cancer. Expert Rev Anticancer Ther 2009;9:413-23.

4. Jones GC, Kehrer JD, Kahn J, et al. Primary Treatment Options for High-Risk/Medically Inoperable Early Stage NSCLC Patients. Clin Lung Cancer 2015;16:413-30.

5. Detterbeck FC, Gibson CJ. Turning gray: the natural history of lung cancer over time. J Thorac Oncol 2008;3:781-92.

6. Tockman MS, Anthonisen NR, Wright EC, et al. Airways obstruction and the risk for lung cancer. Ann Intern Med 1987;106:512-8.

7. de Torres JP, Bastarrika G, Wisnivesky JP, et al. Assessing the relationship between lung cancer risk and emphysema detected on low-dose CT of the chest. Chest 2007;132:1932-8.

8. Skillrud DM, Offord KP, Miller RD. Higher risk of lung cancer in chronic obstructive pulmonary disease. A prospective, matched, controlled study. Ann Intern Med 1986;105:503-7.

9. Mannino DM, Aguayo SM, Petty TL, et al. Low lung function and incident lung cancer in the United States: data From the First National Health and Nutrition Examination Survey follow-up. Arch Intern Med 2003; 163:1475-80.

10. Moro-Sibilot D, Aubert A, Diab S, et al. Comorbidities and Charlson score in resected stage I nonsmall cell lung cancer. Eur Respir J 2005;26:480-6.

11. Young RP, Hopkins RJ, Christmas T, et al. COPD prevalence is increased in lung cancer, independent of age, sex and smoking history. Eur Respir J 2009;34:380-6.

12. Zhai R, Yu X, Shafer A, et al. The impact of coexisting COPD on survival of patients with early-stage non-small cell lung cancer undergoing surgical resection. Chest 2014;145:346-53.

13. Shin S, Park HY, Kim H, et al. Joint effect of airflow limitation and emphysema on postoperative outcomes in early-stage nonsmall cell lung cancer. Eur Respir J 2016;48:1743-50.

14. Yoshida Y, Kage H, Murakawa T, et al. Worse Prognosis for Stage IA Lung Cancer Patients with Smoking History and More Severe Chronic Obstructive Pulmonary Disease. Ann Thorac Cardiovasc Surg 2015;21:194-200.

15. Sekine Y, Yamada Y, Chiyo M, et al. Association of chronic obstructive pulmonary disease and tumor recurrence in patients with stage IA lung cancer after complete resection. Ann Thorac Surg 2007;84:946-50.

16. Donington J, Ferguson M, Mazzone P, et al. American College of Chest Physicians and Society of Thoracic Surgeons consensus statement for evaluation and management for high-risk patients with stage I non-small cell lung cancer. Chest 2012;142:1620-35.

17. NCCN Clinical Practice Guidelines in Oncology. NonSmall Cell Lung Cancer. Available online: https:// www. nccn.org/professionals/physician_gls/pdf/nscl.pdf (Last accessed on May 27th, 2020).

18. McGarry RC, Song G, des Rosiers P, et al. Observationonly management of early stage, medically inoperable lung cancer: poor outcome. Chest 2002;121:1155-8.

19. Wisnivesky JP, Bonomi M, Henschke C, et al. Radiation therapy for the treatment of unresected stage I-II nonsmall cell lung cancer. Chest 2005;128:1461-7.

20. Carter-Harris L. Lung cancer stigma as a barrier to medical help-seeking behavior: Practice implications. J Am Assoc Nurse Pract 2015;27:240-5.

21. Daher M. Cultural beliefs and values in cancer patients. Ann Oncol 2012;23 Suppl 3:66-9.

22. Schneider BJ, Daly ME, Kennedy EB, et al. Stereotactic Body Radiotherapy for Early-Stage Non-Small-Cell Lung Cancer: American Society of Clinical Oncology Endorsement of the American Society for Radiation Oncology Evidence-Based Guideline. J Clin Oncol 2018;36:710-9.

23. Videtic GMM, Donington J, Giuliani M, et al. Stereotactic body radiation therapy for early-stage non-small cell lung cancer: Executive Summary of an ASTRO Evidence-Based Guideline. Pract Radiat Oncol 2017;7:295-301.

24. Miller MR, Hankinson J, Brusasco V, et al. Standardisation of spirometry. Eur Respir J 2005;26:319-38.

25. American Thoracic Society. Single-breath carbon monoxide diffusing capacity (transfer factor). Recommendations for a standard technique--1995 update. Am J Respir Crit Care Med 1995;152:2185-98.

26. Choi JK, Paek D, Lee JO. Normal predictive values of spirometry in Korean population. Tuberc Respir Dis 
2005;58:230-42.

27. Park JO, Choi IS, Park KO. Normal predicted values of single-breath diffusing capacity of the lung in healthy nonsmoking adults. Korean J Intern Med 1986;1:178-84.

28. Harvey BG, Strulovici-Barel Y, Kaner RJ, et al. Risk of COPD with obstruction in active smokers with normal spirometry and reduced diffusion capacity. Eur Respir J 2015;46:1589-97.

29. Goldstraw P, Crowley J, Chansky K, et al. The IASLC Lung Cancer Staging Project: proposals for the revision of the TNM stage groupings in the forthcoming (seventh) edition of the TNM Classification of malignant tumours. J Thorac Oncol 2007;2:706-14.

30. Kim H, Yoo H, Pyo H, et al. Impact Of Underlying Pulmonary Diseases On Treatment Outcomes In EarlyStage Non-Small Cell Lung Cancer Treated With Definitive Radiotherapy. Int J Chron Obstruct Pulmon Dis 2019;14:2273-81.

Cite this article as: Kang N, Shin SH, Noh JM, Kang D, Kim H, Kwon OJ, Pyo H, Ahn YC, Kim HK, Choi YS, Kim J, Zo JI, Shim YM, Cho J, Park HY. Treatment modality and outcomes among early-stage non-small cell lung cancer patients with COPD: a cohort study. J Thorac Dis 2020;12(9):4651-4660. doi: 10.21037/ jtd-20-667
31. Whitson BA, Groth SS, Duval SJ, et al. Surgery for earlystage non-small cell lung cancer: a systematic review of the video-assisted thoracoscopic surgery versus thoracotomy approaches to lobectomy. Ann Thorac Surg 2008;86:200816; discussion 2016-8.

32. Bryant AK, Mundt RC, Sandhu AP, et al. Stereotactic Body Radiation Therapy Versus Surgery for Early Lung Cancer Among US Veterans. Ann Thorac Surg 2018;105:425-31.

33. Cykert S, Dilworth-Anderson P, Monroe MH, et al. Factors associated with decisions to undergo surgery among patients with newly diagnosed early-stage lung cancer. JAMA 2010;303:2368-76.

34. Moser RP, Arndt J, Han PK, et al. Perceptions of cancer as a death sentence: prevalence and consequences. J Health Psychol 2014;19:1518-24.

35. Lange P, Celli B, Agusti A, et al. Lung-Function Trajectories Leading to Chronic Obstructive Pulmonary Disease. N Engl J Med 2015;373:111-22. 


\section{Supplementary}

Table S1 Baseline characteristics of patients with early-stage NSCLC and COPD

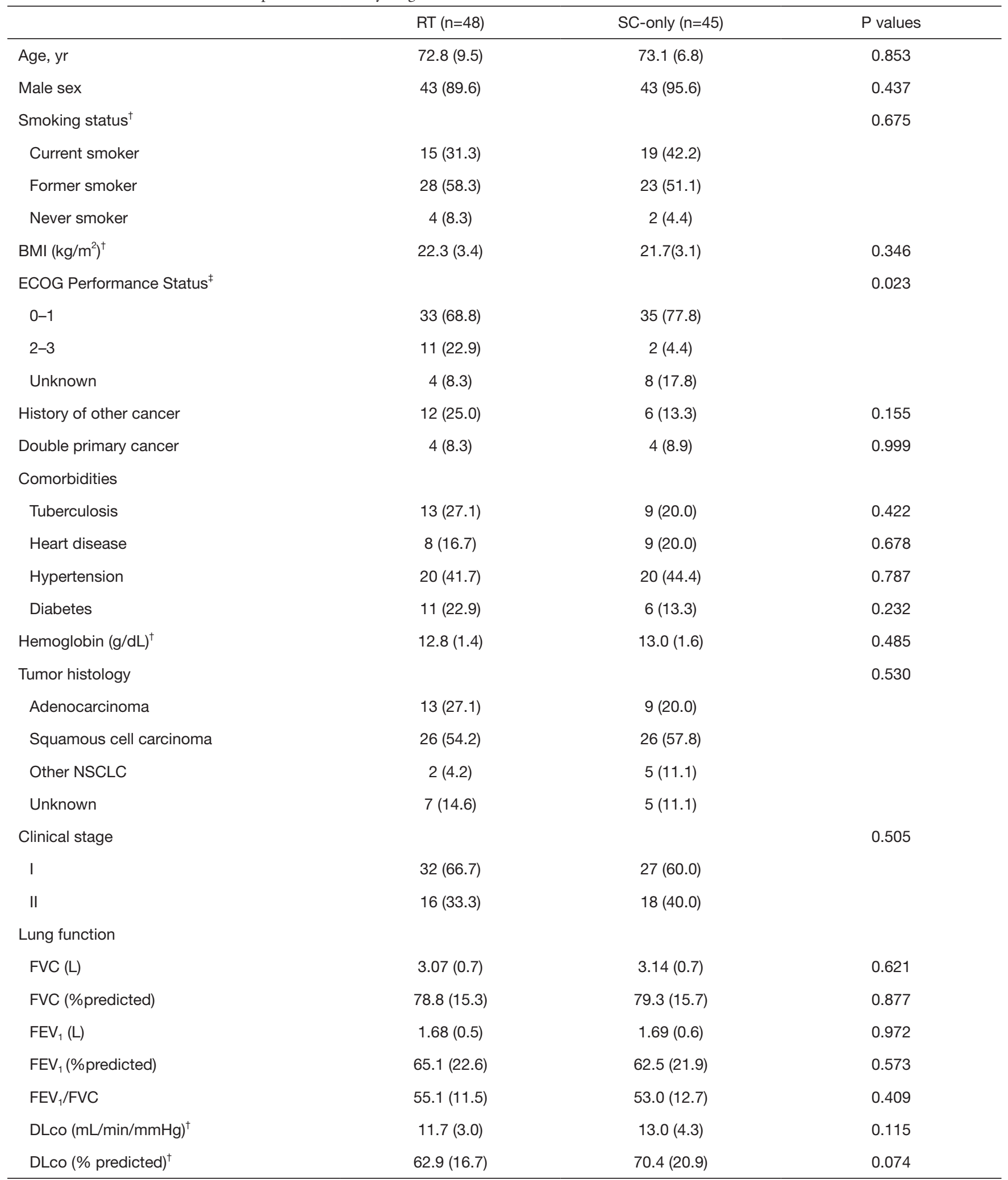

Values are in mean (standard deviation) or number (\%). ${ }^{\dagger}$ The number of patients with missing value was smoking status ( $\left.\mathrm{n}=2\right)$, BMI $(n=3)$, hemoglobin $(n=3)$ and DLco $(n=9)$. ҒPerformance status was based on Eastern Cooperative Oncology Group (ECOG) scale. BMI, body mass index; COPD, chronic obstructive pulmonary disease; DLco, diffusing capacity for carbon monoxide; ECOG PS, Eastern Cooperative Oncology Group Performance Status; FEV 1 , forced expiratory volume in 1 s; FVC, forced vital capacity; NSCLC, non-small cell lung cancer; RT, radiotherapy; SC, supportive care. 
Table S2 Hazard ratios (95\% confidence intervals) for all-cause mortality according to the treatment modality by age group

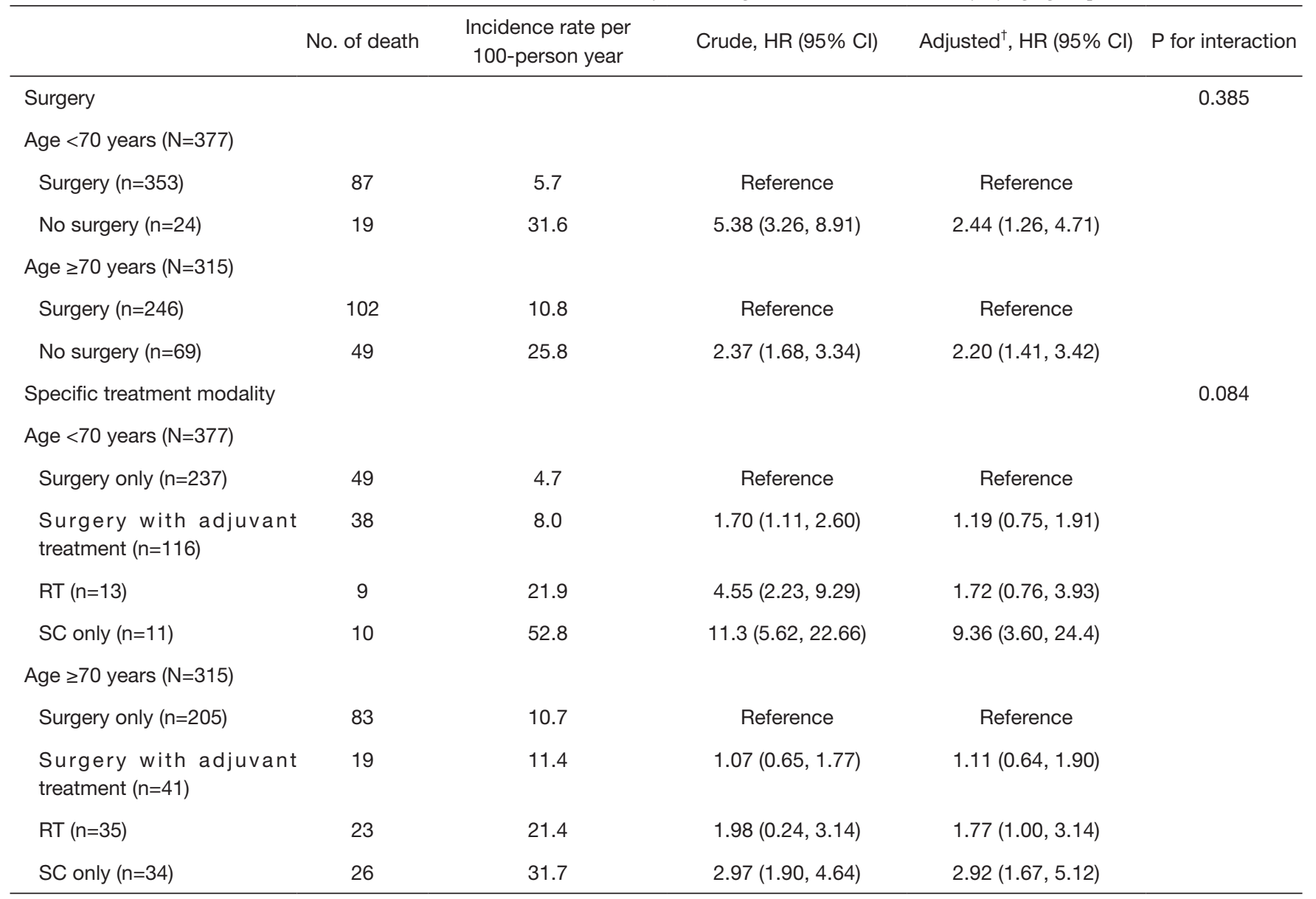

\footnotetext{
${ }^{\dagger}$ Adjusted for sex, smoking status. BMI, performance status, any comorbidity, hemoglobin level, tumor stage, FEV ${ }_{1} \%$ pred and DLco

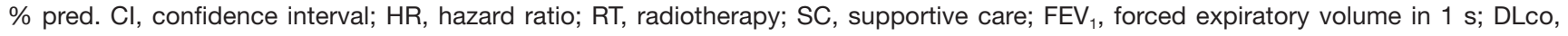
diffusing capacity for carbon monoxide.
} 
Table S3 Hazard ratios (95\% confidence intervals) for all-cause mortality according to the treatment modality among patients with clinical stage I $(\mathrm{N}=455)$

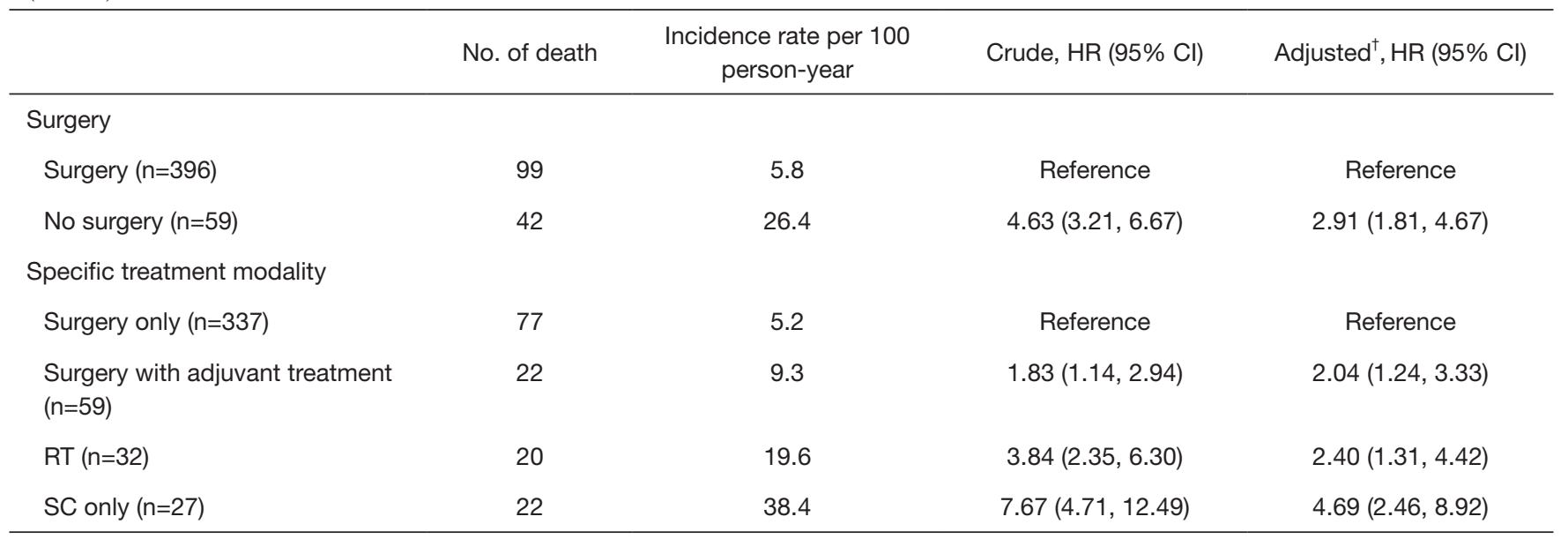

${ }^{\dagger}$ Adjusted for age, sex, smoking status. BMI, performance status, any comorbidity, hemoglobin level, FEV $\%$ pred and DLco \% pred. $\mathrm{Cl}$, confidence interval; HR, hazard ratio; RT, radiotherapy; SC, supportive care; FEV ${ }_{1}$, forced expiratory volume in 1 s; DLco, diffusing capacity for carbon monoxide.

Table S4 Decision making process for NSCLC patients with COPD who did not receive surgical resection

\begin{tabular}{|c|c|c|c|}
\hline & $\mathrm{RT}(\mathrm{n}=48)$ & SC-only $(n=45)$ & $P$ value \\
\hline Patient or families & $13(27.1)$ & $32(71.1)$ & \\
\hline Doctor & 35 (72.9) & $13(28.9)$ & \\
\hline \multicolumn{4}{|c|}{ Main reason(s) for treatment decision ${ }^{\dagger}$} \\
\hline Comorbid disease & $12(25.0)$ & $2(4.4)$ & 0.006 \\
\hline Poor performance state & $2(4.2)$ & $7(15.6)$ & 0.084 \\
\hline Poor lung function & $27(56.3)$ & 7 (15.6) & $<0.001$ \\
\hline Patient and family will & $13(27.1)$ & 33 (73.3) & $<0.001$ \\
\hline
\end{tabular}

The values are in $\mathrm{n}(\%) .{ }^{\dagger}$ Multiple choice questionnaire. NSCLC, non-small cell lung cancer; COPD, chronic obstructive pulmonary disease; $\mathrm{RT}$, radiotherapy; SC, supportive care. 